\title{
Carnets
}

Revue électronique d'études françaises de l'APEF

Deuxième série - 17 | 2019

Lîlle : prisme de la connaissance ou reconnaissance du monde

\section{L'île Non Sachant ou la méconnaissance du monde arthurien}

\section{Emanuele Arioli}

\section{(2) OpenEdition}

Journals

Édition électronique

URL : http://journals.openedition.org/carnets/10007

DOI : $10.4000 /$ carnets. 10007

ISSN : 1646-7698

Éditeur

APEF

Référence électronique

Emanuele Arioli, «L'île Non Sachant ou la méconnaissance du monde arthurien », Carnets [En ligne], Deuxième série - 17 | 2019, mis en ligne le 30 novembre 2019, consulté le 08 janvier 2020. URL : http:// journals.openedition.org/carnets/10007; DOI : 10.4000/carnets.10007

Ce document a été généré automatiquement le 8 janvier 2020.

\section{(c) (i) \&}

Carnets est mis à disposition selon les termes de la licence Creative Commons - Atribution - Pas d'utilisation commerciale 4.0 International. 


\title{
L'île Non Sachant ou la méconnaissance du monde arthurien
}

\author{
Emanuele Arioli
}

1 Un roman du XIII ${ }^{e}$ siècle, bâti sur l'invention d'une île encore vierge, raconte les aventures d'un nouveau héros insulaire qui est censé surpasser tous les chevaliers de la Table Ronde. Il s'agit de Ségurant ou le Chevalier au Dragon, ensemble narratif qui a connu un grand succès à son époque et a circulé de l'Italie à l'Angleterre et de la France à l'Espagne avant de disparaître de la mémoire littéraire ${ }^{1}$. Auparavant inconnu, il se trouvait morcelé dans des manuscrits dispersés dans toute l'Europe: nous l'avons recomposé à partir de tous les fragments et épisodes subsistants. Sa version la plus longue et la plus ancienne, que nous avons appelée " cardinale », a été écrite en langue française en Italie du Nord, peut-être à Venise, entre 1240 et 1279 ; ses prolongements et ses réécritures s'échelonnent du XIII ${ }^{\mathrm{e}}$ au XVI ${ }^{\mathrm{e}}$ siècle $^{2}$.

2 Cet ensemble narratif s'inscrit dans l'univers fictionnel de la légende du roi Arthur, des chevaliers de la Table Ronde et du Graal. Plus précisément, ses modèles principaux sont deux romans - également anonymes - de la première moitié du XIII ${ }^{\mathrm{e}}$ siècle : le Lancelot en prose et le Tristan en prose ${ }^{3}$. Ségurant ou le Chevalier au Dragon peut être défini comme une continuation paraleptique (ou paraquel) de ces deux textes, c'est-à-dire une œuvre qui se déroule dans le même cadre chronologique, mais qui raconte d'autres histoires (Genette, 1982: 243-244). Son originalité tient surtout à l'invention d'une intrigue totalement nouvelle dont le protagoniste est Ségurant, un chevalier naif et gourmand qui va à la cour du roi Arthur plein d'espoir, lorsque deux vilaines fées le lancent à la poursuite d'un dragon illusoire.

3 Cette intrigue s'ouvre par le récit du naufrage des ancêtres de Ségurant et par leurs aventures sur une île déserte - l'île Non Sachant - où, dans une sorte de «robinsonnade » médiévale, ils essayent de refaire le parcours vers la vie civilisée à partir des moyens à leur disposition. Dans le reste du roman, cet espace insulaire est le point de repère du protagoniste dans son parcours de formation à la chevalerie et dans 
sa découverte progressive de l'univers du roi Arthur. C'est à travers le prisme déformant de l'île Non Sachant que les héros perçoivent le monde extérieur et viceversa. Nous proposerons quelques jalons interprétatifs de cet espace insulaire en décryptant l'imaginaire à l'œuvre dans la représentation de cette île encore vierge, en analysant le modèle utopique d'organisation sociale des naufragés et, enfin, en observant comment l'invention de cette l'île structure l'œuvre entière, offrant à son auteur un espace de liberté dans le cadre de la tradition arthurienne.

\section{La représentation et l'imaginaire d'une île « non sachant »}

4 Au début de la « version cardinale » de Ségurant ou le Chevalier au Dragon, Galehaut le Brun le Vieux et son frère Hector le Brun le Vieux - grand-père et grand-oncle du protagoniste - s'embarquent avec quatre marins pour fuir le roi Vertigier qui a usurpé le trône de Logres. Une tempête les fait échouer sur une île qui «était très loin des autres îles et n'était connue de personne ». Cette île inconnue et totalement vierge n'est pas hostile et désertique comme la plupart des espaces insulaires des romans de la Table Ronde (Tristan en prose, L'Histoire du saint-Graal, La Quête du saint-Graal). Au contraire, " elle est extraordinairement riche en bois, rivières, plaines et montagnes ". Cet espace heureux rassemble les caractéristiques constitutives du locus amoenus et, surtout, dans l'optique des héros civilisateurs qui le découvrent, «tout ce qui convient à une bonne ville ».

Quand les naufragés explorent l'île et s'assurent du fait qu'elle est inhabitée, l'un d'eux choisit le toponyme d'Île Non Sachant ainsi que le nom de Port Trouvé pour désigner un port naturel :

Ensuite, ils parcoururent toute l'île et ils découvrirent que la côte était bonne et entourée de hautes falaises. Cherchant bien de tous les côtés, ils constatèrent qu'elle offrait un bon endroit pour accéder à la côte et en repartir. L'un dit à l'autre qu'il s'agissait d'un port extraordinairement beau et bon ; et l'un des marins dit que cette île pouvait être appelée l'île Non Sachant et que le port pouvait être appelé le Port Trouvé. Depuis lors, c'est ainsi qu'ils furent appelés 4 .

6 Le nom « île Non Sachant " peut suggérer son caractère utopique et irréel, féérique et merveilleux. Il se rapproche d'autres toponymes arthuriens - tels que les Lointaines îles ou l'île Perdue ${ }^{5}$ - qui soulignent l'éloignement de l'espace insulaire, à cela près qu'il fait référence à l'idée de connaissance plutôt qu'à la dimension spatiale. L'ancien français permet une double interprétation de "non sachant», qui peut avoir non seulement un sens actif mais aussi passif. L'île Non Sachant serait à la fois sujet et objet de connaissance : elle est « ignorante », puisqu'elle ne connaît pas la civilisation de la terre ferme, et «ignorée », en tant que terra incognita encore inexplorée. Elle est à la fois «à l'état de nature » et «inconnue ». Paradoxalement, l'imposition de ce nom soustrait l'île à l'état sauvage, en marquant sa conquête par l'homme qui dompte et apprivoise la nature. Elle constitue une étape capitale dans le processus de civilisation de l'île, qui, de "non sachant », ne gardera que le nom et le souvenir ${ }^{6}$.

7 Ce récit de colonisation d'une île déserte est probablement issu du croisement de deux schémas narratifs, celui du naufrage à la suite d'une tempête, bien attesté dans la littérature arthurienne, et celui de la fondation d'une ville après un périple. Par rapport aux autres romans arthuriens (L'Histoire du saint-Graal et en moindre mesure $L a$ 
Quête du saint-Graal et le Tristan en prose) où les îles ne sont que des étapes d'un parcours semé d'épreuves, dans Ségurant, les ancêtres du héros éponyme s'établissent sur l'île Non Sachant et y fondent une ville. Au Moyen Âge, les modèles les plus évidents de ce dernier motif sont l'Énéide de Virgile et la conquête de l'Angleterre par Brutus (Historia regum Britanniae de Geoffroy de Monmouth et Roman de Brut de Wace ${ }^{7}$ ). À la différence d'Énée qui combat les Rutules ou de Brutus qui affronte des géants, les ancêtres de Ségurant ne doivent pas entreprendre de guerre. Le nouvel espace est inhabité : il n'est pas conquis, mais il est découvert à la suite d'un naufrage. Cette invention narrative d'une île sauvage colonisée incarne probablement les rêves des cultures méditerranéennes au temps des croisades. Elle pourrait révéler une «idéologie coloniale» avant la lettre, celle des républiques maritimes avec leurs ambitions sur la mer Méditerranéenne et en particulier de Venise au XIII ${ }^{\mathrm{e}}$ siècle avec son hégémonie sur les îles grecques.

\section{L'utopie d'une « robinsonnade » médiévale}

8 Ségurant ou le Chevalier au Dragon décrit l'organisation sociale des naufragés sur l'île, leur vie matérielle, leur retour progressif à la civilisation. Il s'inspire sans doute d'un «imaginaire colonial» avant la lettre, qui n'est pas sans analogie avec celui que déploiera quelques siècles plus tard - et bien plus largement - Robinson Crusoé. Dans Ségurant, les naufragés ont tout de suite le pressentiment qu'ils resteront sur l'île jusqu'à leur mort. Galehaut incite alors ses compagnons à organiser leur vie commune de la meilleure façon possible :

Quand le bateau se heurta à la côte, il se brisa entièrement. Ceux qui se trouvaient à l'intérieur - et qui étaient presque morts - sortirent à l'extérieur sur la terre sèche. Quand le jour se leva beau et clair et le vent se fut calmé, ils transportèrent au rivage leur équipement. Galehaut prit donc la parole en disant: Seigneurs, il nous faudra rester ici pour toute notre vie et mourir. Faisons cela au mieux que nous pourrons. Prenons le bois de ce bateau et les clous : construisons un refuge où nous pourrons rester les nuits'. Ils s'accordèrent tous sur cela ${ }^{8}$.

9 Les naufragés recréent alors une microsociété qui continue d'être régie par les normes en vigueur dans leur terre d'origine. Le contact avec la sauvagerie de l'île ne cause pas de bouleversements sociaux dans leur petit groupe: en conservant la hiérarchie sociale, les chevaliers - membres de l'aristocratie - imposent leur domination sociale sur leurs compagnons d'infortune. Ainsi les naufragés se partagent-ils leurs tâches avec une division clairement hiérarchique; les deux chevaliers chassent tandis que les quatre marins cueillent des fruits et servent spontanément les premiers :

Maintenant, sachez, seigneurs, que les quatre marins étaient tellement bons qu'ils servaient les deux vaillants chevaliers comme s'ils étaient leurs seigneurs liges. Les deux frères chevaliers se fabriquèrent un arc chacun et se façonnèrent des flèches, en faisant les pointes avec les clous du bateau. Et je vous dis ouvertement qu'ils commencèrent à prendre des oiseaux et des bêtes sauvages en quantité. Telle fut leur nourriture tant qu'ils restèrent sur l'île. Ils avaient abondamment à manger de la viande et des pommes sauvages que les quatre marins allaient chercher dans les bois de l'île, et je vous dis clairement que les deux chevaliers devinrent si gros et vigoureux en peu de temps que, si leurs proches les avaient vus, ils ne les auraient pas reconnus. Chaque jour, les deux chevaliers allaient chasser et prenaient de grands oiseaux et de grosses bêtes. Les quatre marins allaient cueillir des pommes, préparaient les repas aux deux chevaliers et les servaient au mieux qu'ils pouvaient ${ }^{9}$. 
10 alors que les naufragés confectionnent tous ensemble des récipients pour cuire leurs aliments. Ils reviennent ainsi à une alimentation plus civilisée, malgré l'absence du pain, si essentiel à la table dans l'Occident médiéval (Le Goff et Vidal-Naquet, 1974 ; Le Goff, 1982).

11 L'activité première sur l'île demeure la chasse. Les oiseaux et le gibier sont tellement abondants - explique le narrateur - que les chevaliers deviennent tellement gros que personne ne pourrait les reconnaître! Une aventure de chasse marque symboliquement l'appropriation de l'espace sauvage. Sur une petite montagne, les naufragés voient une bête d'une taille extraordinaire; Galehaut tire sur elle une flèche qui la blesse ; elle commence à fuir, mais, près de l'eau, elle se laisse atteindre et tuer :

Un jour, lorsqu'ils traversaient lîle ensemble tous les six, ils trouvèrent une bête d'une taille extraordinaire qui se dressait en haut d'une petite montagne. Quand ils la virent, ils allèrent vers elle, mais la bête était tellement fière qu'elle ne daigna pas fuir. Au contraire, elle attendit que Galehaut, qui précédait les autres, la blessa d'une flèche au milieu de la poitrine. Quand la bête sentit le coup, elle se mit à fuir. Hector, qui la suivait de près, la blessa d'une autre flèche. Ainsi s'enfuyait-elle et les chevaliers l'atteignirent près de l'eau et, là, ils la prirent et la tuèrent. Puis les quatre marins l'apportèrent à leur refuge ${ }^{10}$.

Cette bête joue le rôle d'une sorte de génie du terroir (genius loci), présence primitive et surnaturelle qui, dans les traditions populaires et les récits littéraires, hante les terres vierges $^{11}$. Sa mise à mort accompagne par sa valeur rituelle le processus de colonisation. naufragés sur l'île Non Sachant, le récit est lacunaire. Notre analyse codicologique révèle que le manuscrit Arsenal 5229 - le seul témoin de cette partie du récit - a perdu un feuillet. Ainsi, sans transition, le lecteur trouve les naufragés au beau milieu d'un tournoi à Salisbury, sans qu'on puisse en déduire comme ils ont pu quitter l'île Non Sachant. Le feuillet perdu dévoilait-il de quelle manière les naufragés quittaient leur île ? Il est permis de le penser, mais il se peut également que l'auteur ait ménagé une ellipse temporelle pour éviter de devoir résoudre cette difficulté narrative. Il se peut également que la lacune soit plus étendue : ce témoin manuscrit est postérieur de plus d'un siècle au récit d'origine ${ }^{12}$.

Quoi qu'il en soit, le récit revient à l'île Non Sachant quelques épisodes après pour raconter la jeunesse de Ségurant : l'île Non Sachant n'est plus une île sauvage, mais un espace bâti et civilisé où s'élèvent des palais, des maisons et même une église. Encore sans transition, la microsociété primordiale s'est transformée en société urbaine et chrétienne. Nos recherches dans les archives et les bibliothèques, qui ont permis de retrouver d'autres fragments, ne nous ont malheureusement pas conduit à retrouver le feuillet manquant : l'issue de cette "robinsonnade » médiévale demeure à ce jour sans solution.

\section{Le destin d'un héros insulaire}

Grâce à l'invention de l'île Non Sachant, le romancier médiéval a voulu donner au protagoniste une éducation à l'écart, loin de la cour arthurienne, sans doute sur le modèle de Lancelot, qui a grandi sous les eaux d'un lac enchanté, et de Perceval, qui a 
été élevé par sa mère au milieu d'une forêt. Comme le Perceval de Chrétien de Troyes et le Lancelot en prose, Ségurant ou le Chevalier au Dragon raconte la formation à la chevalerie du protagoniste, sa découverte progressive du monde arthurien avec ses coutumes et ses règles.

16 Au début du récit, l'île est le miroir du héros: le sens premier de «nonsachant » est bien celui d'« ingénu », de "naïf », d'« ignorant ». Son parcours initiatique est scandé par plusieurs étapes, comme dans un roman d'apprentissage. Après son adoubement, Ségurant quitte la maison familiale à l'insu de son père qui l'en aurait empêché . À travers cette transgression, le héros adolescent se dérobe à l'autorité paternelle et se livre seul au monde. Si l'espace clos de l'île représente l'enfance, la terre ferme - où s'étendent les espaces ouverts et illimités des forêts et des landes - peut figurer le monde des adultes et de l'aventure. Quand le héros a prouvé sa valeur sur la terre ferme, par la joute victorieuse contre son oncle, il peut revenir sur son île natale et être accueilli dans la communauté des adultes.

S'éloignant de son île, le héros perd son insouciance initiale et acquiert, d'épreuve en épreuve, une confiance excessive en lui-même. Il quitte ainsi une deuxième fois son île afin de prouver sa valeur à la cour du roi Arthur. Au tournoi de Winchester, il se fait remarquer par des exploits admirables, mais, pour éloigner ce héros qui pourrait être un allié redoutable du roi Arthur, la fée Morgane et l'enchanteresse Sibylle invoquent un diable qui prend l'apparence d'un dragon et sème la terreur sur le lieu du tournoi. Ségurant franchit un mur de feu et se lance à la poursuite du monstre, en succombant ainsi au sortilège des deux mauvaises fées. Il tend tous les efforts de son corps et de son esprit vers cet objet fantomatique qu'il ne pourra jamais atteindre. Le dragon-diable est « une chose spirituelle », comme l'explique le narrateur, et ne peut pas être tué :

Mais cette pensée était trompeuse, car il ne pourrait tuer le dragon en aucune manière, puisque celui-ci était une chose spirituelle. Et l'esprit, bon ou mauvais qu'il soit, ne meurt jamais : seule la chair meurt, ainsi comme Merlin le témoigne ouvertement quand il dit que, bien que sa chair fût morte et pourrie, son esprit était vivant ${ }^{13}$.

18 Sans nouvelles de Ségurant, ses compatriotes de l'île Non Sachant partent à sa recherche, tandis que la cour arthurienne, abusée par les machinations perfides de Morgane, se demande si ce chevalier a réellement existé ou s'il ne s'agissait que d'un mirage.

19 Le destin de Ségurant est sans doute marqué par la fatalité de son origine insulaire. Dans les textes littéraires médiévaux, l'île n'est pas seulement le lieu d'origine d'objets merveilleux (épées, armures, anneaux magiques), mais également d'êtres extraordinaires, comme si cet espace clos était propice à la conservation d'une généalogie fabuleuse (Dubost, 1991: I, 293-294). Doté d'une stature merveilleuse et d'une grande ambition ${ }^{14}$, Ségurant paraît pouvoir être reconnu pour le meilleur chevalier de sa génération, mais, obnubilé par l'enchantement qui l'oblige à suivre un mirage, il finit par être oublié.

20 À la fin de la plus ancienne version, probablement inachevée, le héros poursuit encore son dragon illusoire. Les divers continuateurs et remanieurs ont voulu pallier les imperfections de ce héros inaccompli. Dans les autres versions, Ségurant part pour la croisade, devient un roi de la Terre sainte ou succombe après ses batailles victorieuses contre les Sarrasins. Sa poursuite du dragon, qui traverse de nombreux manuscrits, 
serait vouée à demeurer inachevée, si un continuateur n'avait décidé d'en finir avec le monstre en lui donnant la mort ${ }^{15}$. héros et un nouveau texte dans l'archipel de la littérature arthurienne. Il a pu déployer l'histoire d'un chevalier insulaire qui a grandi à l'écart de la cour arthurienne, sur le modèle de Lancelot et de Perceval, et qui découvre le monde extérieur en s'éloignant du berceau de son enfance. Le toponyme d'île Non Sachant suggérait déjà la centralité de la question de la connaissance du monde : si l'île est d'abord le lieu «non sachant » - à l'état sauvage et inconnu - que les colonisateurs découvrent, il devient ensuite l'espace à partir duquel le protagoniste pose son regard «nonsachant » - insouciant et ingénu - sur le monde.

Sur cette île vierge s'inscrit comme sur une page blanche la vie d'un nouveau héros qui méconnaît le monde. Sa naïveté initiale permettait de faire le récit complet de sa découverte de la chevalerie, en conduisant le lecteur d'une île éloignée au cœur même de la cour arthurienne. Mais l'isolement de l'enfance annonçait aussi un autre isolement, celui de l'oubli auquel Ségurant est voué. Sorti d'une île inconnue, le protagoniste disparaît aussi mystérieusement qu'il est apparu et il est dès lors considéré par ses compagnons de fiction comme un être fictif, mirage produit par un enchantement.

Le choix de ce héros insulaire destiné à l'oubli offrait à l'auteur une plus grande autonomie et liberté par rapport aux romans précédents auxquels Ségurant ou le Chevalier au Dragon empruntait l'univers romanesque. Dans cette invention, le romancier médiéval a sans doute été guidé par le projet de greffer un nouveau texte sur le corpus de la littérature arthurienne sans pourtant contredire les romans précédents, qui se situaient dans le même cadre chronologique et qui ignoraient ce chevalier nouveau venu. Par le biais de l'île Non Sachant, il a propulsé dans l'univers du roi Arthur un héros qui est censé surpasser Lancelot et Tristan, tout en assurant la cohérence de son ouvrage avec les récits antérieurs. De tels jeux d'illusion et d'oubli ont sans doute favorisé le destin d'évanescence de Ségurant et marqué de leur fatalité l'ensemble narratif dont il est le protagoniste.

\section{BIBLIOGRAPHIE}

ARIOLI, Emanuele (2016). Ségurant ou le Chevalier au Dragon : roman arthurien inédit (XIII XV siècles), Histoire littéraire de la France (t. 45). Paris : Académie des Inscriptions et Belles-Lettres. ARIOLI, Emanuele (2019a). Ségurant ou le Chevalier au Dragon : version cardinale (tome 1). Paris : Champion. 
ARIOLI, Emanuele (2019b). Ségurant ou le Chevalier au Dragon : versions complémentaires et alternatives (tome 2). Paris : Champion.

ARIOLI, Emanuele (2019c). Ségurant ou le Chevalier au Dragon (XIII $\mathrm{XV}^{e}$ siècles) : étude d'un roman arthurien retrouvé. Paris : Champion (« Nouvelle Bibliothèque du Moyen Âge »).

BAUMGARTNER, Emmanuèle (1995). « Brut et les ancêtres d'Arthur », PRIS-MA, bulletin de liaison de l'équipe de recherche sur la littérature d'imagination du Moyen Âge, 11, pp. 139-148.

COHEN, Jeffrey Jerome (2000). The Postcolonial Middle Ages. New York: Palgrave.

Cosman, Madeleine Pelner (1966). The Education of the Hero in Arthurian Romance. Chapel Hill : University of North Carolina Press.

DUBOST, Francis (1991). Aspects fantastiques de la littérature narrative médiévale (XII XIII ${ }^{e}$ siècles) :

l'Autre, l'Ailleurs, l'Autrefois. Paris : Champion.

FRAPPIER, Jean (1976). « La Mort Galehaut », in Id., Histoire, mythes et symboles. Études de littérature

française. Genève : Droz, pp. 137-147.

GAUNT, Simon (2009). « Can the Middle Ages be Postcolonial ? ", Comparative Literature, 61, $\mathrm{n}^{\circ} 2$, pp. 160-176.

GENETTE, Gérard (1982). Palimpsestes : la littérature au second degré. Paris: Seuil.

INGHAM, Patricia Clare et Warren, Michelle R. (2003). Postcolonial Moves: medieval through modern. New York: Palgrave.

LE GOFF, Jacques (1970). «Culture ecclésiastique et culture folklorique au Moyen Âge », in Luigi De Rosa (org.), Ricerche storiche ed economiche in memoria di Corrado Barbagallo. Napoli : Edizioni scientifiche italiane, 1970, t. II, pp. 51-90. In Jacques le Goff, Un Autre Moyen Âge. Paris : Gallimard, 1999, pp. 229-268.

LE GOFF, Jacques et Vidal-Naquet, Pierre (1974). « Levi-Strauss en Brocéliande : esquisse pour une analyse d'un roman courtois », Critique, 325, pp. 543-571. In Jacques Le Goff, Un Autre Moyen Âge. Paris : Gallimard, 1999, pp. 581-614.

LE GOFF, Jacques (1982), «Quelques remarques sur les codes vestimentaire et alimentaire dans Érec et Énide », in La Chanson de geste et le mythe carolingien, Mélanges René Louis. Saint-Père-sousVézelay : Musée archéologique régional, II, pp. 1243-1258. « Codes vestimentaire et alimentaire dans Érec et Énide ", in Jacques Le Goff, Un Autre Moyen Âge. Paris : Gallimard, 1999, pp. 615-634.

LECOUTEuX, Claude (1996). Démons et génies du terroir au Moyen Âge. Paris : Imago.

MICHA, Alexandre (1978-1983). Lancelot, roman en prose du XIII ${ }^{e}$ siècle. Genève : Droz.

MORA-LEBRUN, Francine (1994). L'« Énéide » médiévale et la naissance du roman. Paris : PUF.

UHLIG, Marion (2014). «Quand 'Postcolonial' et 'Global' riment avec 'Médiéval' : sur quelques approches théoriques anglo-saxonnes ", Perspectives médiévales, 35 (mis en ligne le 1 janvier 2014), [consulté le 21 septembre 2019]. http://journals.openedition.org/peme/4400.

\section{NOTES}

1. Une allusion à son protagoniste, sous le nom de Severause le Brewse, figure dans le roman anglais Le Morte Darthur de Thomas Malory (1485); d'autres allusions à Ségurant le Brun se trouvent dans les libros de caballerías espagnols Tristán de Leonís (1501) et Amadis de Gaule (1508), 
ainsi que dans quelques poèmes chevaleresques de la Renaissance italienne (Girone il Cortese et l' Avarchide de Luigi Alamanni, I Quattro primi canti del Lancilotto d'Erasmo da Valvasone). Sur la fortune littéraire de cet ensemble narratif, voir Arioli, 2016 et Arioli, 2019c.

2. Ce roman vient de paraître dans les «Classiques français du Moyen Âge » (Arioli, 2019a et Arioli, 2019b).

3. En reprenant à ces deux œuvres beaucoup de personnages et de chronotopes, le narrateur fait place à des aventures prétendument oubliées dans ses modèles. Il promet de raconter « plusieurs aventures et plusieurs exploits de Tristan et de Palamède qui n'ont pas été traduits dans les autres livres» («maintes belles aventures et maint grant fait de Tristan et de Palamedes qui es autres livres ne furent translatez », ép. XVII de la « version cardinale », ms. Arsenal 5229, fol. 99ra) ou encore «d'autres aventures qui ne sont pas racontées dans les autres livres» («d'autres aventures qui ne sont pas es autres livres devisees ", ép. xxxIv, fol. 136va), puisque "plusieurs récits sur lui [Lancelot] n'ont pas été traduits du latin au français » (« maint comptes de lui furent lessiez a translater de latin en françoys », ép. xvi, fol. 96vb). Voir Arioli, 2019a : 331-337.

4. «Et aprés cerchierent l'isle de totes pars, et le bois et la marine troverent ilz auques bon et cloz tout environ de bon murs, dont li mariniers le cerchierent bien de toutes pars et trouverent qu'il avoit a merveilles bonne entree et bonne yssue, dont il dist li uns a l'autre que a merveilles estoit biaux et bons cestui port; et li uns des mariniers dist que celle ysle pouoit estre appellee l'Isle Non Sachant et li port pouoit estre appellez li Port Trouvez, dont ainsi furent puis appellez » (« version cardinale », épisode I, ms. Arsenal 5229, fol. 23ra ; Arioli, 2019a : 94).

5. Les Lointaines îles sont la patrie de Galehaut, aussi bien dans le Lancelot que dans le Tristan en prose, tandis que l'île Perdue est le lieu où Galehaut séjourne avec son ami dans le Lancelot en prose.

6. Comme Emmanuèle Baumgartner l'écrit à propos du Roman de Brut, «civiliser (...) c'est essentiellement nommer. C'est imposer à un univers informe, sans repères, sans ancrages temporels ou spatiaux, des noms qui tout à la fois relient l'homme à son passé et qui signifient par eux-mêmes le cours du temps et le sens de l'histoire » (Baumgartner, 1995 : 141).

7. L'histoire de Brutus s'inspire largement de celle d'Énée (Mora-Lebrun, 1994 : 56-71).

8. "Quant la barge fery, elle se debrisa toute; et cilz dedens, qui presque mort estoient, yssirent hors a seiche terre. Et quant li jours fu venuz clers et biaux et li airs fut acoisiez, ilz eschapperent tout leur harnoiz a seiche terre. Et lors parolle Galehot et dit: 'Seignours, yci nous [22va] estuet mourir et estre en vie tout nostre aage. Or le faison bien au miex que nous pourron. Prenons les fust de ceste barge et les cloz et faison un retret ou nous puissons herbergier les nuiz.' Et ils s'accordent a ce tuit. » (« version cardinale », épisode I, ms. Arsenal 5229, fol. 22rb-22va ; Arioli, 2019a: 93).

9. «Or saichiez, seignours, que li quatre mariniers furent tant debonnaires qu'ilz servoient et honoroient les deux bons chevaliers ainsi comme s'ilz feussent leur lige seignour. Li duy bons chevaliers freres firent chascun un arc et pourchacerent d'avoir saietes et y firent les fers des clouz de la barge, dont je vous di appertement qu'ilz commencerent a prendre oyseaux et bestes a grant plenté. Tot ce fu leur viande, tant qu'ilz furent en celle isle. Assés avoient a mengier char et pomes sauvages, mez de pain n'avoient ilz point. Ilz firent vaissiaux et les sçorent bien cuire, et puis firent dedens cervoises de pometes sauvages que li quatre [22vb] aloient cueillant parmy le boiz de l'isle, dont je vous di appertement que li duy chevaliers devindrent si gras et si membrus en si pou de temps qu'ilz ne fussent congneuz de leurs voisins qui a donc les veissent par aucune maniere du monde. Li duy bons chevaliers aloient en chace chascun jour et prenoient les grans oyseaux et les grans bestes, et li . IIII. mariniers aloient cerchant pommettes et appareilloient leurs mengier aux deux chevaliers et les servoient de tout leur pouoir " ("version cardinale ", épisode I, ms. Arsenal 5229, fol. 22va-22vb; Arioli, 2019a : 93-94).

10. «Un jour avint qu'ilz aloient tuit .vi. ensemble parmy l'isle, si trouverent une beste qui de grant corps estoit a merveilles, qui estoit dessus une petite montaigne. Et quant ilz la virent, ilz 
se vindrent tuit. VI. celle part, mes la beste estoit tant orguilleuse qu'elle ne deigna fouir, ains attendi tant que messire Galehot, qui aloit devant les autres, la fery d'une saiete parmy le pis. Et quant la beste senti adonc le cop, elle se mist en fuie ; et messire Hector, qui tres devant se mist, la fery d'une saiette. Si s'enfuioit la beste, et li chevaliers l'ataignent auques pres de l'eaue et illec [23ra] la pristrent et l'occistrent, et li quatre mariniers l'enporterent a leur recet " («version cardinale », épisode I, ms. Arsenal 5229, fol. 22vb-23ra ; Arioli, 2019a : 94).

11. Les génies du terroir apparaissent parfois sous la forme d'animaux dans les textes littéraires (Lecouteux, 1995). Le triomphe de l'homme sur un génie du terroir a aussi été transposé dans l'hagiographie sous une forme christianisée: le saint - souvent un évêque évangélisateur triomphe d'un dragon-diable, comme la Grand'Goule, la Gargouille ou le Graouilly (Le Goff, 1970).

12. Plusieurs fragments et manuscrits incomplets de la "version cardinale " datent du XIII ${ }^{e}$ siècle.

13. «Mez sa pensee li failly, car ce dragon ne pourroit il occire en nulle maniere du monde, car il estoit chose esperituele. Et li esperiz, bon ou mauvez qu'i soit, ne muert jamez : la char meurt seulement, ainsi com Merlin le tesmoigne appertement ça en arriere, quant il dist que, combien que sa char fust morte et pourrie, son esperit estoit vif... » (" version cardinale », épisode xxx, ms. Arsenal 5229, fol. 126va ; Arioli, 2019a : 272).

14. Ségurant peut rappeler un autre héros insulaire de la légende arthurienne qui atteint la gloire avant de déchoir: Galehaut, le seigneur des Lointaines Îles. Après avoir soumis trente royaumes, Galehaut renonce à la conquête de Logres, voit toutes ses forteresses s'écrouler en une seule nuit, puis meurt de chagrin à cause de sa passion pour Lancelot (Micha, 1978-1983 : I, 10-17, 388 ; Frappier, 1976).

15. La mort du dragon est racontée dans le premier épisode de la "version alternative » de Londres-Turin, conservée par le ms. Londres, British Library, Add. 36673 et par le premier tome des manuscrits Turin, Biblioteca Nazionale Universitaria, L.I.7-9 (Arioli, 2019 b : 207-212).

\section{RÉSUMÉS}

Un roman du XIII ${ }^{\mathrm{e}}$ siècle, jusqu'à présent inconnu, a inventé un nouvel espace insulaire pour proposer une nouvelle version de la légende du roi Arthur. Les ancêtres du héros éponyme Ségurant - font naufrage et s'établissent sur une île encore vierge qu'ils appellent l'île Non Sachant. Quant au protagoniste, il abandonne son île natale pour aller à la cour du roi Arthur, où deux vilaines fées l'obligent à poursuivre un dragon illusoire. Tout au long du récit, l'île Non Sachant est un prisme déformant à travers lequel les héros insulaires perçoivent le monde extérieur et vice-versa. L'article propose de décrypter l'imaginaire à l'œuvre dans la représentation de cette île, l'organisation sociale de ses habitants ainsi que les raisons de ce choix insulaire offrant à l'auteur une marge de liberté dans la tradition de la littérature arthurienne.

A thirteenth century Arthurian Romance, so far unknown, has invented a new island to propose a new version of the legend of King Arthur. The protagonist's ancestors shipwrecked and settled on a wild island they called Non Sachant Island. As for the protagonist, he leaves his native island to go to King Arthur's court, where two bad fairies force him to pursue an illusory dragon. Throughout the story, Non Sachant Island is a distorting prism through which the islanders perceive the outside world and vice versa. This article decode the imaginary in the representation of this island, the social organization of its inhabitants as well as the reasons for 
this insular choice offering the author a margin of freedom in the tradition of Arthurian literature.

INDEX

Keywords : Middle Ages, Arthurian Romance, island, prism, imaginary

Mots-clés : Moyen Âge, roman arthurien, île, prisme, imaginaire

\section{AUTEUR}

EMANUELE ARIOLI

Université Polytechnique Hauts-de-France

emanuelearioli[at]gmail.com 